\title{
Panorama sobre Educación Sexual Integral, deseo y prospectiva de embarazo entre adolescentes de Querétaro
}

\section{A panorama on Comprehensive Sex Education, desire and prospective pregnancy among adolescents in Querétaro, Mexico}

DOI: https://doi.org/10.32870/dse.v0i21.633

\author{
Úrsula Gayou-Esteva* \\ María Elena Meza-de-Luna** \\ Sergio Noriega-Vivanco*** \\ Luz Graciela Vázquez-Bautista****
}

Resumen

Se realizó una investigación cuantitativa con 321 estudiantes de secundaria y dos docentes, para reconocer el deseo y la prospectiva de embarazo entre adolescentes, identificar las características de la Educación Sexual Integral (ESI) que se desarrolla en la escuela y localizar las principales fuentes de información para las y los adolescentes en temas de sexualidades. Entre el estudiantado que espera tener hijos/as (75.4\%), la edad elegida para reproducirse fue de 25.57 años, pero $4 \%$ de la muestra considera para ello edades compatibles con el Embarazo Adolescente (EA). La principal fuente de información sobre sexualidades fue la madre para $46.7 \%$. Solo $12.5 \%$ reportó que todos los contenidos de ESI se han abordado en clases. El tema más tratado fue el uso correcto del condón (95\%) y el menos, fue el placer (35.6\%). Las mujeres tienen más información que los hombres sobre los tipos de violencia y cómo evitarlos (78.4\% hombresvs.88.0\% mujeres; $X^{2}=5.11, \mathrm{GL}=1, \mathrm{p}=0.02$ ). Conduimos que la ESl aún es deficiente y reproduce estereotipos de género. Se recomienda redoblar esfuerzos para que la población adolescente pueda tomar decisiones informadas.

Palabras clave: deseo de embarazo - educación sexual integral - embarazo en la adolescencia - fuentes de información sobre sexualidades - perspectiva de género.

\section{Abstract}

Quantitative research was conducted with 321 high school students and two teachers, to recognize desire and prospective pregnancy among adolescents, identify the characteristics of Comprehensive Sex Education

\footnotetext{
* Doctorante en Estudios Interdisciplinarios sobre Pensamiento. Coordinadora de Proyectos en la Asociación Civil IIPSIS Investigación e Intervención Psicosocial, (www.iipsis.mx).Querétaro. México.ursulagayou@gmail.com

** Doctora en Psicología Social. Miembro del Sistema Nacional de Investigadores (SNI). Universidad Autónoma de Querétaro. Coordinadora del Observatorio Ciudadano de Seguridad del Municipio de Querétaro. México. mezamariel@gmail.com

*** Maestrante en Psicología Clínica. Profesor en el Diplomado de Intervención en Crisis, Universidad Autónoma de Querétaro. México. noriega.sv@gmail.com

*** Socióloga. Interés profesional: temas relacionados con la identidad dentro de los grupos sociales y en fenómenos como la pobreza, la marginación y la desigualdad. Universidad Autónoma de Querétaro. México. singthesorrow.2@gmail.com
} 
(CSE) that take place in the school, and locate the main sources of information for adolescents on sexuality issues. Among the students who expect to have children (75.4\%), the age chosen to reproduce was 25.57 , but $4 \%$ of the sample considered doing it at ages compatible with Adolescent Pregnancy (AP). The main source of information on sexuality was the mother (46.7\%). Only $12.5 \%$ of the students reported that all the CSE content had been addressed in class. The topic discussed most often was the correct use of the condom (95\%), and the least discussed was pleasure (35.6\%). Women are more informed about types of violence and how to avoid them $(78.4 \%$ men vs. $88.0 \%$ women, $\mathrm{X} 2=5.11, \mathrm{GL}=1, \mathrm{p}=0.02)$. We concluded that the CSE is still deficient and reproduces gender stereotypes, and recommended redoubling efforts to help adolescents make informed decisions.

Key words: desire for pregnancy - comprehensive sexual education - pregnancy in adolescence - sources of information about sexualities - gender perspective.

\section{Introducción}

En la actualidad el embarazo en la adolescencia (EA) se ha convertido en un problema complejo. Esto quiere decir, por un lado, que no siempre el EA fue considerado un problema (Lagarde, 2015; Stern, 2003) y la dimensión de complejidad alude a que, a contracorriente de la opinión popular sobre el fenómeno (que generalmente se enfoca en casos particulares), en realidad este depende solo en una mínima parte de los encuentros sexuales desprotegidos o mal protegidos entre mujeres adolescentes y hombres de cualquier edad con capacidad reproductiva (Secretaría de Educación Pública - SEP, 2012). Detrás del EA, en su carácter de problema complejo, existe un entramado propiciatorio de factores sociales, culturales, religiosos, económicos, políticos y educativos (Family Care International, 2008; SEP, 2012), lo cual resulta necesario investigar a partir de acercamientos interdisciplinarios y críticos que trasciendan la misión biopolítica (Fernández, 2013) de controlar la natalidad de las personas menores de 20 años y, en su lugar, apunten como alternativa a fortalecer la agenda pública en materia de acceso a Educación Sexual Integral (ESI) para que "cada embarazo sea deseado, cada parto sea sin riesgos y cada persona joven alcance su pleno desarrollo" (Fondo de Población de las Naciones Unidas - UNFPA), 2017: s.p.).

La ESI es una propuesta educativa internacionalmente probada, basada en un enfoque de Derechos Humanos y orientada a promover conocimientos y habilidades para la toma de decisiones responsables sobre sexualidad, salud y relaciones personales (Rojas et al., 2017). Ofrecer ESI de calidad es un compromiso todavía pendiente del Estado mexicano con niñas, niños y adolescentes, y las carencias formativas que se desprenden de las limitantes en la incorporación curricular y manejo de esta materia, constituyen un importante factor de riesgo frente al EA.

En esa línea de intereses, la presente investigación se propone aportar hallazgos respecto a ciertos elementos de reconocida influencia en el EA, a través de una aproximación con perspectiva de género, al deseo y prospectiva de embarazo que sostienen las y los adolescentes; la actualización de información sobre sus fuentes de consulta principales cuando tienen dudas 
sobre sexualidades y la caracterización de la ESI que se aborda en su contexto escolar, para perfilar avances y retos todavía pendientes en la materia en el entorno local y nacional.

\section{Embarazo en la adolescencia en contexto: falta de oportunidades y negación de derechos}

Tanto a nivel mundial, como en nuestro país, las estadísticas de fecundidad y estudios en la materia evidencian que se convierten en madres y padres a temprana edad principalmente jóvenes que sufren pobreza ${ }^{1}$ (UNFPA, 2017), presentan de antemano desapego o falta de acceso al estudio (Family Care International, 2008), poseen escasas oportunidades de desarrollo profesional a futuro (Pacheco-Sánchez, 2016; Posada, 2014), y manifiestan deficiencias formativas en temas de Educación Sexual Integral (ESI) que limitan sus posibilidades de tomar decisiones informadas sobre su sexualidad y su reproducción (Rojas et al., 2017). Esto implica que muchas mujeres y algunos varones adolescentes, que no han tenido acceso al ejercicio de sus propios derechos, están teniendo que hacer frente a la experiencia de maternar o paternar - en ocasiones sin desearlo- en situaciones de abandono institucional y/o familiar, y con pocas posibilidades reales de acceder a una vida digna y plena para sí mismos/as y sus hijos/as (Altamirano, 2017).

En la agenda pública, los esfuerzos de prevención del EA están orientados por un discurso generalizante que atribuye a estos embarazos consecuencias negativas inherentes para quienes lo enfrentan directamente y a gran escala, para el desarrollo del país ${ }^{2}$ (UNFPA, 2013). Sin embargo, estudios sobre los factores políticos y macroeconómicos implicados en la alta incidencia de EA, han subrayado que la vulnerabilidad no proviene del fenómeno sino, en todo caso, el EA es

propiciado por las condiciones de vulnerabilidad social en que llegan a encontrarse muchas adolescentes, como consecuencia de condiciones estructurales que limitan su acceso a instituciones y redes de apoyo que [...] permiten a otras jóvenes superar situaciones y problemas..." (Stern, 2003: 739, ver también Family Care International, 2008; García-Hernández, 2014; Posada, 2014retraso de la edad para contraer matrimonio, desempleo, Baja escolaridad...).

Esta consideración debe llevarnos a introducir matices, porque claramente no es lo mismo embarazarse siendo muy joven pero contando con redes de apoyo familiar para la crianza, con la posibilidad de estudiar y teniendo garantizado el sustento de ese hijo/a, que pasar por el mismo proceso sabiendo que las oportunidades de estudiar han quedado truncadas, vivien-

1 En países con alta incidencia de EA, la tasa de fecundidad de las adolescentes que residen en $20 \%$ de los hogares más pobres es casi tres veces superior a la de aquellas de $20 \%$ de los hogares más ricos (UNFPA, 2017).

2 A gran escala, estos EA favorecen la reproducción de una pobreza que les antecede, a veces por varias generaciones y cuya reproducción podría ser frenada de manera más directa por políticas anticorrupción y de redistribución de la riqueza. 
do rechazo, soledad y hasta expulsión del núcleo familiar ${ }^{3}$ (Fernández, 1998; Pacheco-Sánchez, 2016; Posada, 2014; Stern, 2003).

Además, existe el factor diferencial del deseo de embarazo o la ausencia de este. En resistencia a la mayoría de las campañas que apuntan a prevenir el EA a partir de mensajes centrados en el riesgo (Family Care International, 2008), la última Encuesta Nacional de la Dinámica Demográfica en México (ENADID, 2014) reiteró el hallazgo de que una importante mayoría de estos embarazos se declaran deseados (82\%) e incluso planeados (69\%) por las adolescentes ${ }^{4}$ (citado en Consejo Nacional de Población - Conapo, 2016). Esta realidad, poco atendida, se contrapone con el discurso contemporáneo imperante que explica el fenómeno como producto de la impulsividad o la irresponsabilidad. En ese sentido, cabe preguntarnos por qué no hemos promovido indagaciones más empáticas que, sin anticiparse a censurar un posible deseo de embarazo, nos permitan identificar en qué medida estos deseos convergen con condiciones de autonomía simbólica y con un contexto familiar facilitador que les permita seguir siendo hijas hasta que alcancen una edad compatible con la autonomía plena (Fernández, 1998).

La importancia que reviste la articulación entre maternidad y deseo de embarazo de las mujeres está llegando tarde a las discusiones políticas sobre el tema en México y en otros países de América Latina (Amuchástegui, Flores y Aldaz, 2015; Rocha Sánchez, 2015). Sigue estando muy extendida la noción de que las mujeres, porque biológicamente tienen el potencial de ser madres, están provistas de un instinto natural que, acompasado con su desarrollo físico, las conduce a reproducirse y así alcanzar la plenitud de su feminidad (Lagarde, 2015). Esta visión obtura la posibilidad de pensar el deseo - y otros determinantes subjetivos - en el campo de la maternidad en toda su complejidad: mujeres que desean ser madres y otras que no, madres que no hubieran deseado serlo y otras que sí, con muchas variantes intermedias y posibilidades de transición. En cambio, por una desafortunada transliteración entre instinto y deseo, se pretendería que las mujeres adultas siempre deseen ser madres (porque su instinto las lleva a ello) y las adolescentes (en tanto mujeres inacabadas) nunca podrían desearlo porque su instinto pertenece a su cuerpo y no a su subjetividad. Así, en la intersección de dos condiciones culturales de opresión: ser mujer y ser adolescente(CDHDF, 2016; Fernández, 1998), se excluye del imaginario social y deviene más o menos impensable que algunas mujeres adolescentes tengan el deseo de ser madres a temprana edad. Sus embarazos se atribuyen al descuido, el arrebato, la ignorancia y hasta al oportunismo.

3 “Cuando hay recursos, la pareja está presente o no existen problemas familiares acuciantes, el embarazo es percibido como no problemático. Aunque éste sea accidental y provoque [...] desconcierto, entre otras emociones, también puede suponer afirmación de la propia identidad, reconocimiento social y acercamiento al mundo adulto" (Pacheco-Sánchez, 2016: 57).

4 Actualmente solo existen estudios exploratorios como el de Quiroz et al. (2014), sobre el deseo de embarazo en hombres muy jóvenes. Sin embargo, en cualquier análisis que aborde el embarazo, deben ser consideradas con mucha seriedad las relaciones de poder y la diferencia biológica entre hombres y mujeres (Figueroa, 2005). Por ello, el deseo de embarazo de los adolescentes deberá ser tratado aparte, en futuros estudios.

Diólopos 
No obstante, sin menoscabo de la importancia que reviste dar cabida al deseo de tener un hijo/a, en el análisis de la causalidad del fenómeno del EA debemos hacer un alto para señalar que, deseados o no, estos embarazos siguen encuadrados en un contexto que no promueve ni acompaña el surgimiento de proyectos alternativos de vida y de realización personal para los y las jóvenes, más allá de la reproducción(Altamirano, 2017), lo que convierte al embarazo, no en una opción, sino en una falta de opciones (Posada, 2014: 7). Además de que, como dice Fernández (1998), para que la maternidad pueda considerarse una elección, deben existir condiciones materiales, psíquicas, institucionales y morales, tanto para ejercer una maternidad gozosa como para optar por no ser madre. En la mayor parte de México, todavía no hemos podido ofrecerles eso a las adolescentes.

\section{Educación sexual integral y perspectiva de género: información para decidir y justicia social}

Enraizados patrones culturales sobre lo que se espera de las personas debido a su sexo biológico, tampoco han favorecido que se matice el impacto que tienen los procesos asociados a la reproducción en el cuerpo de las mujeres, para que esta diferencia biológica no derive en injusticia. El enfoque biologicista que tradicionalmente ha primado en los abordajes del EA, nos dice Posada (2014: 7), ha dificultado la valoración "de los riesgos psicosociales, económicos y culturales que pueden no provocar la muerte física de la adolescente, sino comprometer el disfrute de una vida digna y hacerla profundamente infeliz". En lugar de promover la corresponsabilidad de los hombres en la crianza, los cuidados y la educación de los hijos e hijas, algunos mensajes sobre el tema siguen apelando a naturalizar el exceso de tareas en estos ámbitos, con su consecuente sobrecarga emocional, como atributos normales propios de la experiencia de ser madre (Lagarde, 2015).

Por mucho tiempo, la educación sexual y los esfuerzos de prevención del EA estuvieron dirigidos únicamente a las mujeres (UNFPA, 2013) y esas disposiciones políticas continúan teniendo presencia en el imaginario social (Quiroz, Atienzo, Campero y Suárez-López, 2014). Investigaciones recientes han documentado que los adolescentes varones no están desarrollando suficiente conciencia del vínculo entre su propia sexualidad y la posibilidad de procrear (Figueroa Perea y Sánchez, 2000; INSP, 2015), lo que determina que con frecuencia asuman posturas de escasa implicación frente a eventuales embarazos. Perciben el EA como algo "malo" pero no fatal para sus vidas, suelen no sentirse responsables de un embarazo en la pareja, llegando a considerarse incluso como una "opción" para ellos la huida ante la paternidad (Quiroz et al., 2014). A diferencia de sus compañeras, no es común que modifiquen su forma de vida, siguen estudiando y saliendo con sus amigos (SEP, 2012).

Los condicionamientos tradicionales de género no solo distribuyen de manera injusta la responsabilidad que asumen mujeres y hombres frente a un EA, sino que tienen una participa- 
ción importante en la alta prevalencia del fenómeno: las adolescentes todavía conviven con la idea de que de ellas se espera que sean madres, esposas y amas de casa, y llegan a sentir que un embarazo es la vía para realizarse como mujeres y ser reconocidas socialmente como tales (Perrotta, 2018). Los varones son socializados para asumir que su masculinidad depende de su capacidad de procrear y proveer, y para ellos, asumir la condición de padre de familia puede representar una salida de la infancia y la adquisición de los emblemas de la virilidad (INSP, 2015).

Somos parte de una época en la que el sistema sexo-género, en los términos antes descritos, está sufriendo fuertes reconfiguraciones (INSP, 2015). Muchas mujeres ya no se suscriben a los ideales tradicionales y muestran mayor autodeterminación respecto a su sexualidad y su reproducción, pero la deconstrucción de la masculinidad hegemónica —en parte por ser más tardía y en parte por implicar el cuestionamiento de privilegios - es todavía muy incipiente (Díaz et al., 2018). Esto implica un factor de riesgo muy importante para la salud y el bienestar de los propios hombres, sus parejas y sus relaciones en general, en tanto las representaciones asociadas a este modelo de masculinidad validan el abuso de poder y excluyen las prácticas de cuidado. Así, "se fomenta que los hombres afirmen su virilidad asumiendo riesgos, reafirmando su valentía, tolerando el dolor, siendo un sostén independiente y teniendo múltiples parejas sexuales" (UNFPA, 2013: 47). Sin embargo, la vigencia de estos valores también incrementa las posibilidades de reproducirse a temprana edad y ello continua teniendo un impacto especialmente duro para las mujeres a quienes embarazan (Díaz et al., 2018; UNFPA, 2013). Por estas razones, entre las iniciativas de estudio y prevención del EA se vuelve indispensable trabajar con perspectiva de género, y se torna urgente desarrollar aproximaciones de ESI que incluyan a los adolescentes como actores igualmente protagónicos que las mujeres (Figueroa, 2005).

La ESI, señalan Rojas et al. (2017: 20), es una propuesta educativa internacionalmente probada y orientada a mejorar "conocimientos, actitudes y habilidades en siete ámbitos: el género, la salud sexual y reproductiva, la ciudadanía sexual, el placer, la violencia, la diversidad y las relaciones". La misma fuente recupera la experiencia en otros contextos y puntualiza que este tipo de abordaje pedagógico de las sexualidades favorece la adquisición de conocimientos en cuanto a Salud Sexual y Reproductiva (SSR) y reduce las prácticas de riesgo:

La ESI no fomenta la actividad sexual, sino que aplaza la edad de inicio de vida sexual, reduce el número de parejas sexuales y mejora las prácticas de protección contra ITS, con lo cual ha demostrado ser una estrategia efectiva para reducir los riesgos asociados con el VIH y aumentar la autoeficacia para el uso de condón. Además de representar un elemento central en la prevención de las ITS y el embarazo no deseado, los currículos de la ESI incluyen componentes que influyen en las actitudes, prácticas y resultados asociados con el ejercicio más amplio de derechos, ciudadanía y relaciones interpersonales en general (2017: 20). 
A pesar de todas las bondades que esta propuesta educativa pueda representar para la vida, la salud y el desarrollo de las personas, y de que actualmente exista un marco legal que obliga al Estado mexicano a garantizar que niñas, niños y adolescentes tengan acceso a ella, ${ }^{5}$ la introducción de la ESI en el Sistema Educativo continúa enfrentando fuertes resistencias (SEP, 2012). A partir de la Reforma Integral de la Educación Básica de 2011, se dispuso que en todos los niveles y grados de educación básica se trabajarían competencias para la vida, valores enfocados a la diversidad, igualdad de género, salud, prevención de violencia y Derechos Humanos (Rosales-Mendoza y Salinas-Quiroz, 2017). Sin embargo, el reporte ofrecido en 2015 por la Declaración Ministerial "Prevenir con Educación Pública" deja ver que los avances en este aspecto no son nada alentadores, "hay deficiencias importantes en la evaluación de los programas educativos para determinar el grado de implementación de la EIS" (Save the Children, 2016:16) y ninguno de los criterios que contempla la propuesta ha sido evaluado como "bien" u "óptimamente" integrado en el currículo y materiales didácticos.

En un estudio derivado del Programa de Becas de Apoyo a la Educación Básica de Madres Jóvenes y Jóvenes Embarazadas (Promajoven) la propia SEP (2012: 92) admite que el abordaje pedagógico de las sexualidades en la mayoría de las escuelas de México "se limita a describir anatómica y fisiológicamente los aparatos reproductivos, prevalece un discurso de índole moral con base en un ideal de constitución de pareja adulta heterosexual establecida con fines reproductivos".

Otro obstáculo para el adecuado cumplimiento de las políticas públicas en materia de ESI, dice también la SEP (2012), han sido los propios gobiernos locales de algunos estados, entre ellos, Querétaro. Recientemente, en ocasión del inicio del ciclo 2018-2019 en esta entidad, circuló en distintos medios de opinión pública la noticia de que solo nueve de 537 secundarias públicas "eligieron", a través de sus Consejos Técnicos, libros que incluyen ESI abierta y explícita (Ochoa, 2018). Otorgar un carácter opcional a la impartición de ESI contraviene la legislación en la materia y limita seriamente el acceso que puedan tener las y los adolescentes al ejercicio informado y responsable de sus Derechos Sexuales y Derechos Reproductivos (CDHDF, 2016; Díaz et al., 2018).

Teniendo en cuenta especialmente: 1) la importancia de conocer qué tan extendido está el deseo y la prospectiva de embarazo entre las y los jóvenes, con miras a desarrollar esfuerzos educativos más sensibles a sus necesidades, 2) la pertinencia de saber qué papel asignan las juventudes al contexto escolar, cuando se trata de informarse sobre temas de sexualidades, y 3) los antecedentes que apuntan a que la ESI está siendo deficientemente implementada a

5 La Ley General de los Derechos de Niños, Niñas y Adolescentes, en su artículo 58, señala que la educación tendrá entre sus fines: “Promover la educación sexual integral conforme a su edad, el desarrollo evolutivo, cognoscitivo y madurez, de las niñas, niños y adolescentes que le permitan a niñas, niños y adolescentes ejercer de manera informada y responsable sus derechos [...]" (Congreso General de los Estados Unidos Mexicanos, 2014). 
nivel nacional y censurada a nivel local, y la correlativa necesidad de conocer las características reales de la ESI de la que se hace partícipes a las y los jóvenes; desarrollamos una investigación con estudiantes y profesorado encargado de impartir tópicos de sexualidades en una secundaria pública del municipio de Querétaro. Nos interesa abonar conocimiento contextualizado y actual sobre estas cuestiones, para inspirar nuevas reflexiones interdisciplinarias en materia de EA, con perspectiva de género, juventudes y Derechos Humanos. Asimismo, pretendemos que este conocimiento potencie la capacidad de problematizar el fenómeno, abandonando los enfoques de riesgo, que parten de contenidos que los simplifican con generalizaciones y que son moralizantes.

\section{Método e instrumentos}

Se realizó un estudio de corte cuantitativo con estudiantes y profesorado, a manera de diagnóstico, para diseñar una iniciativa de intervención orientada a promover entre la población estudiantil el desarrollo de factores de protección frente a conductas y situaciones de riesgo relacionadas con la sexualidad y la propensión al consumo de sustancias. ${ }^{6}$

Por oportunidad, la investigación se llevó a cabo en una secundaria pública del municipio de Querétaro, que ofrece educación a casi 600 jóvenes, presenta rezago educativo (Instituto Mexicano para la Competitividad, A.C., 2017) y se ubica en una zona de cierta vulnerabilidad social. El Área Geoestadística Básica (AGEB) en que se encuentra ubicada la escuela, tiene un grado medio de marginación urbana y un grado bajo de rezago social urbano (Poder Ejecutivo del Estado de Querétaro, 2019).

Con el estudiantado se utilizó un cuestionario multipropósito para indagar factores de riesgo y de protección frente a conductas y situaciones que podrían tener un impacto nocivo para sus vidas. Asimismo, se recabaron datos sociodemográficos básicos. El instrumento incluyó ocho ítems enfocados en EA y ESI; siete de los cuales fueron retomados de investigaciones recientes realizadas con población mexicana en rangos de edad y situaciones contextuales similares a las de nuestro grupo de interés (Atienzo, Campero, Lozada y Herrera, 2014; Quiroz et al., 2014; Rojas et al., 2017). Con base en dichos ítems, se exploraron: 1) aspiraciones educativas, 2) motivaciones atribuidas al deseo de un embarazo en la adolescencia, 3) deseos de tener hijos/as y edad ideal para ello, 4) principal fuente de consultas sobre sexualidad, y 5) contenidos sobre ESI abordados en el contexto escolar. En este escrito presentaremos lo concerniente a los tres últimos temas.

Los ítems que exploran deseos de tener hijos/as y edad considerada ideal para ello (" $¿ \mathrm{Te}$ gustaría tener hijos/as?", “¿A qué edad te gustaría tener hijos/as?") fueron recuperados de una investigación afín, hecha por Atienzo et al. (2014) con adolescentes de Ciudad de México y Morelos.

6 Presentar los pormenores de la intervención, rebasa los objetivos de este trabajo. 
Para indagar la fuente principal de consultas de las y los jóvenes sobre sexualidades, se planteó la siguiente pregunta: “¿A quién es más probable que consultes si tienes alguna duda o problema relacionado con temas de sexualidad?". Las opciones de respuesta fueron: papá, mamá, profesor/a, médico/a o enfermero/a en un Centro de Salud, amistades, fuentes de internet y otra persona o fuente.

Por otra parte, con el propósito de obtener información sobre los contenidos de ESI que han sido recomendados internacionalmente para que se aborden durante la educación primaria y secundaria, se adaptó una matriz con 15 temáticas que pueden ser agrupadas en tres dimensiones (Salud Sexual y Reproductiva (SSR), Autoeficiencia y, Derechos y relaciones). Esto se retomó de la investigación de Rojas et al. (2017), que originalmente contiene 24 temas repartidos en estas mismas tres dimensiones. La adaptación se hizo en función de acortar el instrumento sin renunciar a los contenidos y, en algunos casos, se englobaron dos o más temas en una categoría nueva, más amplia (p.e., "Respeto de la diversidad por discapacidad", "Respeto de la diversidad por estatus de VIH y sida", "Respeto de la diversidad por orientación sexual" y "Respeto de la diversidad por origen étnico", se conjuntaron para formar "Respeto de la diversidad por origen étnico, por orientación sexual, por estatus de VIH o por discapacidad"). Asimismo, se consideró pertinente agregar el tema: "la masturbación", dentro de la categoría SSR y "estereotipos de género", en Derechos y relaciones.

Tabla 1: Matriz de dimensiones y temas de ESI

\begin{tabular}{|c|c|c|}
\hline SSR & Autoeficacia & Derechos y relaciones \\
\hline $\begin{array}{l}\text { La pubertad, cambios físicos y } \\
\text { procesos emocionales. }\end{array}$ & $\begin{array}{l}\text { El uso correcto del condón o de } \\
\text { otros métodos anticonceptivos. }\end{array}$ & Igualdad y equidad de género. \\
\hline $\begin{array}{l}\text { Cómo protegerse para prevenir } \\
\text { un embarazo. }\end{array}$ & $\begin{array}{l}\text { Dónde y cómo conseguir condo- } \\
\text { nes u otros métodos anticoncep- } \\
\text { tivos. }\end{array}$ & $\begin{array}{l}\text { Tipos de violencia (física, sexual, } \\
\text { psicológica, etc.) y cómo evitarlos } \\
\text { en las relaciones. }\end{array}$ \\
\hline $\begin{array}{l}\text { Cómo protegerse para prevenir el } \\
\text { VIH y otras infecciones de trasmi- } \\
\text { sión sexual. }\end{array}$ & $\begin{array}{l}\text { Cómo evitar situaciones que pu- } \\
\text { dieran llevarte a tener relaciones } \\
\text { sexuales que no deseas o sin pro- } \\
\text { tección. }\end{array}$ & $\begin{array}{l}\text { Respeto de la diversidad por } \\
\text { origen étnico, por orientación } \\
\text { sexual, por estatus de VIH o por } \\
\text { discapacidad. }\end{array}$ \\
\hline \multirow[t]{3}{*}{ La masturbación. } & Acceso a servicios de salud & El placer. \\
\hline & $\begin{array}{l}\text { Cómo hablar con tu pareja sobre } \\
\text { usar condón. }\end{array}$ & $\begin{array}{l}\text { Los derechos sexuales y repro- } \\
\text { ductivos. }\end{array}$ \\
\hline & & Estereotipos de género \\
\hline
\end{tabular}

Nota: Adaptada de "Educación sexual integral: Cobertura, homogeneidad, integralidad y continuidad en escuelas de México" (Rojas, de Castro, Villalobos, Allen-Leigh, Braverman-Bronstein y Uribe, 2017).

El acercamiento con el personal docente responsable de impartir tópicos de sexualidades en la misma institución, se realizó mediante entrevistas semiestructuradas centradas en cono- 
cer sus visiones respecto a los problemas que de manera general enfrentan actualmente las y los jóvenes; el EA —incluyendo su causalidad, la recurrencia con que se presentan casos en la escuela y cómo se procede institucionalmente frente a ellos-; el alcance que tiene la formación en sexualidades en la vida del estudiantado; la metodología que utilizan para impartir dichos temas; y la profundidad con que son abordados los contenidos de ESI en las clases que imparten. Para este escrito retomamos solo la última cuestión, que se indagó con base en una escala numérica entre cinco y uno, sobre la misma matriz adaptada de Rojas et al. (2017) y empleada para el instrumento de estudiantes.

\section{Procedimiento y análisis}

Presentamos los instrumentos y la propuesta de levantamiento de datos al director de la escuela. Nos autorizó hacer contacto con el personal docente y se definieron fechas y horarios de aplicación del cuestionario con los diferentes grupos escolares.

Mediando el respectivo consentimiento por parte de ambos docentes, las entrevistas se realizaron de manera individual y en un contexto de privacidad. En ambos casos se autorizó la grabación de voz y se utilizó la matriz de ESI impresa para facilitar la evaluación numérica.

El levantamiento de cuestionarios con el estudiantado comenzó con la lectura del consentimiento informado a cada grupo. Se resolvieron dudas y se comprobó que los y las estudiantes participaban voluntariamente antes de proceder a la autoaplicación del cuestionario. La asistente de investigación resolvió las dudas que surgieron al responder el instrumento. Se obtuvieron permisos de las y los tutores para la aplicación del instrumento.

El análisis cuantitativo se realizó con estadística descriptiva, tablas de contingencia, pruebas de chi cuadrado para estudiar las posibles diferencias en función del género. Todos los análisis estadísticos se hicieron con el programa IBM SPSS Statistics versión 22.

\section{Resultados y discusión}

Respondieron el cuestionario 321 adolescentes de entre 12 y 16 años $(M=13.4, D S=0.80)$, de los cuales $52.6 \%$ fueron hombres (169 hombres y 152 mujeres). La situación económica de sus familias fue autodefinida como: mala o muy mala $1.9 \%$, regular $31.1 \%$, buena o muy buena $64.2 \%$, y en $2.8 \%$ de los casos no se obtuvo respuesta. $88.8 \%$ de estudiantes vive con su madre; $74.8 \%$ con su padre; ${ }^{7} 81.9 \%$ con hermanos/as; $30.5 \%$ comparte residencia con abuelos/as; $0.9 \%$ con madrastra y $4.4 \%$ con padrastro.

Paralelamente, se obtuvo información de parte de dos docentes (un hombre y una mujer) sobre los contenidos de ESI que abordan en sus clases.

$770.1 \%$ forman parte de una familia nuclear, es decir, cohabitan con su padre y su madre.

Diálo@os sobre Educación año 11 | número 21 | julio-diciembre 2020 | ISSN 2007-2171 


\section{Deseo y prospectiva de embarazo}

La mayor parte (75.4\%) de las y los adolescentes quiere tener hijos algún día (78.5\% de los hombres y $72 \%$ de las mujeres; $X^{2}=1.79, G L=1, p=0.18$ ). La mayoría espera tenerlos entre los 25 y 29 años, sin haber diferencia significativa entre los géneros para la distribución de la edad en esta expectativa (Gráfica 1; $X^{2}=14.57, \mathrm{GL}=21, \mathrm{p}=0.84$ ). De hecho, en promedio esperan tener su primer hijo o hija a los 25.57 años $( \pm 3.77)$, sin que exista una diferencia estadísticamente significativa entre hombres y mujeres $(t=-0.236, G L=229, p=0.81$; donde la expectativa de edad para la paternidad es en promedio $25.52 \pm 3.96$ años y para la maternidad es $25.63 \pm 3.55$ años). En una investigación afín, realizada por Atienzo et al. (2014), la edad deseable para tener hijos/ as reportada por jóvenes de tercero de secundaria y bachillerato en Morelos y la Ciudad de México, fue de 27 años en promedio.

Es importante destacar que $6 \%$ de los jóvenes y $2 \%$ de las jóvenes aportaron edades menores a los 20 años como aquellas personalmente deseables para tener un hijo/a. Esto incrementa las posibilidades de que vivan un EA deseado, y posiblemente hasta planeado (Atienzo et al., 2014); pero no necesariamente decidido en condiciones de autonomía.

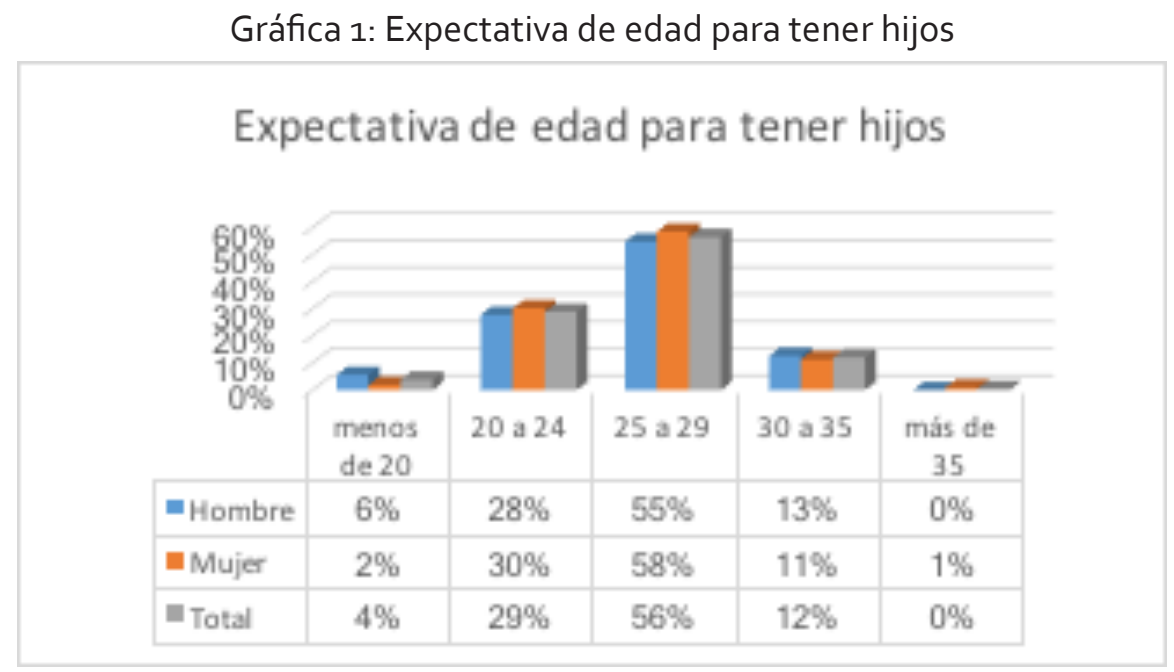

Fuente: datos de investigación propia. Querétaro, 2017.

\section{Fuentes de información sobre sexualidades}

Encontramos diferencia estadísticamente significativa entre el orden de preferencia que establecen los y las adolescentes respecto a sus fuentes de información sobre sexualidades $\left(X^{2}=\right.$ $58.16, G L=6, p=0.000$ ). La principal fuente en ambos casos es la madre (Gráfica 2). Sin embargo, mientras que los hombres, después de la mamá recurren más frecuentemente al padre, fuentes de internet, centros de salud, amistades y profesores; las mujeres recurren con mucha 
menor frecuencia a fuentes de información alterna a la madre, como: amistades, centros de salud, padre, internet y profesores.

En un estudio previo sobre EA, desarrollado en Querétaro por Carrillo et al. (2012), se encontró que tanto las mujeres como los hombres jóvenes conversan sobre sexualidades preferentemente con su madre (55.9\% para las primeras y $42.2 \%$ para los segundos), con amistades ( $21.4 \%$ y $26.3 \%$ ), con personal de salud ( $11.1 \%$ y $19.4 \%$ ) y con profesores/as de su escuela (9.7\% y $8.9 \%$ ). Nuestra investigación confirma el papel protagónico de las madres en la educación sexual y la relegada presencia que está teniendo el profesorado como fuente en el mismo tema, superada incluso por consultas en internet.

En relación con lo anterior, resulta llamativo que en la investigación de Carrillo et al. (2012), la escuela sí haya sido identificada como fuente primaria de obtención de conocimientos sobre métodos anticonceptivos (seguida de la familia y el centro de salud). Datos más recientes de la Agencia Save the Children (2016: 6) en México, también revelan que a nivel nacional "más del $53 \%$ de las y los adolescentes identifica a su escuela como el lugar en donde recauda mayor información sobre sexualidad". Posiblemente, la baja referencia al personal docente como fuente principal de consulta sobre sexualidades esté más relacionada con factores como la confianza, el trato personalizado y la confidencialidad, que con la mera capacidad de brindar información. En este sentido, a continuación, se presentan algunos pormenores de la ESI que están recibiendo las y los adolescentes en sus escuelas.

Gráfica 2: Personas e instituciones a quienes se recurre para consultas sobre sexualidad ¿A quién es más probable que consultes si tienes alguna duda o problema relacionado con temas de sexualidad?

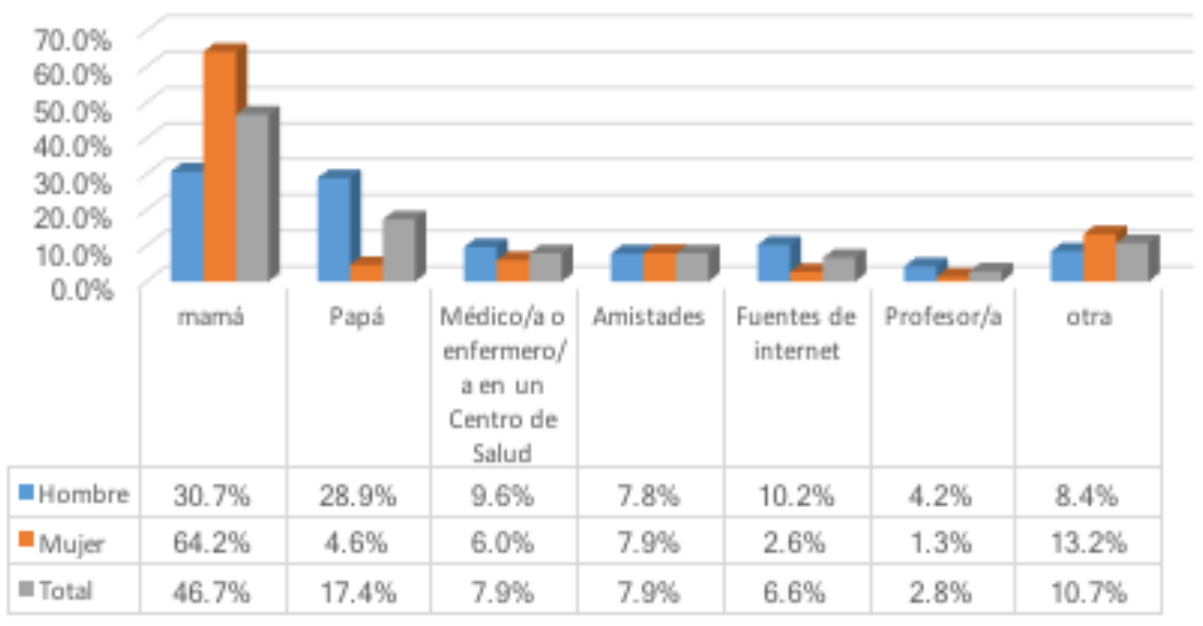

Fuente: investigación propia. Querétaro, 2017.

Diálo@os sobre Educación 


\section{Educación Sexual Integral}

En el curso de la primaria y/o secundaria, los tres principales temas de ESI que los y las adolescentes han recibido son: 1) uso correcto del condón o de otros métodos anticonceptivos (95\%); 2) pubertad, cambios físicos y procesos emocionales (91.2\%); y 3 ) igualdad y equidad de género (88.6\%) (Gráfica 3). En contraparte, de los que menos han recibido información son: 1) el placer (35.6\%), 2) cómo hablar con tu pareja sobre usar condón (37.2\%) y 3) la masturbación (47.6\%).

De la ponderación hecha por el personal docente responsable de la ESI sobre los mismos temas, en función de la profundidad con que los abordan en sus clases, se obtuvieron resultados similares. Los temas que tratan con mayor énfasis son: 1) el respeto de la diversidad por origen étnico, por orientación sexual, por estatus de $\mathrm{VIH}$ o por discapacidad (5 puntos en una escala de 5), 2) cómo protegerse para prevenir un embarazo (5 puntos), 3) el uso correcto del condón o de otros métodos anticonceptivos (4.5 puntos) y 4) la igualdad y equidad de género (4.5 puntos). Por otro lado, aquellos que abordan con menor profundidad son: 1) cómo evitar situaciones que pudieran llevar a tener relaciones sexuales que no desea o sin protección (1.5 puntos), 2) la masturbación (1.5 puntos), 3) cómo hablar con su pareja sobre usar condón (2 puntos) y 4 ) el placer ( 2 puntos) (véase tabla 3 ). 
Gráfica 3: Temas de Educación Sexual Integral

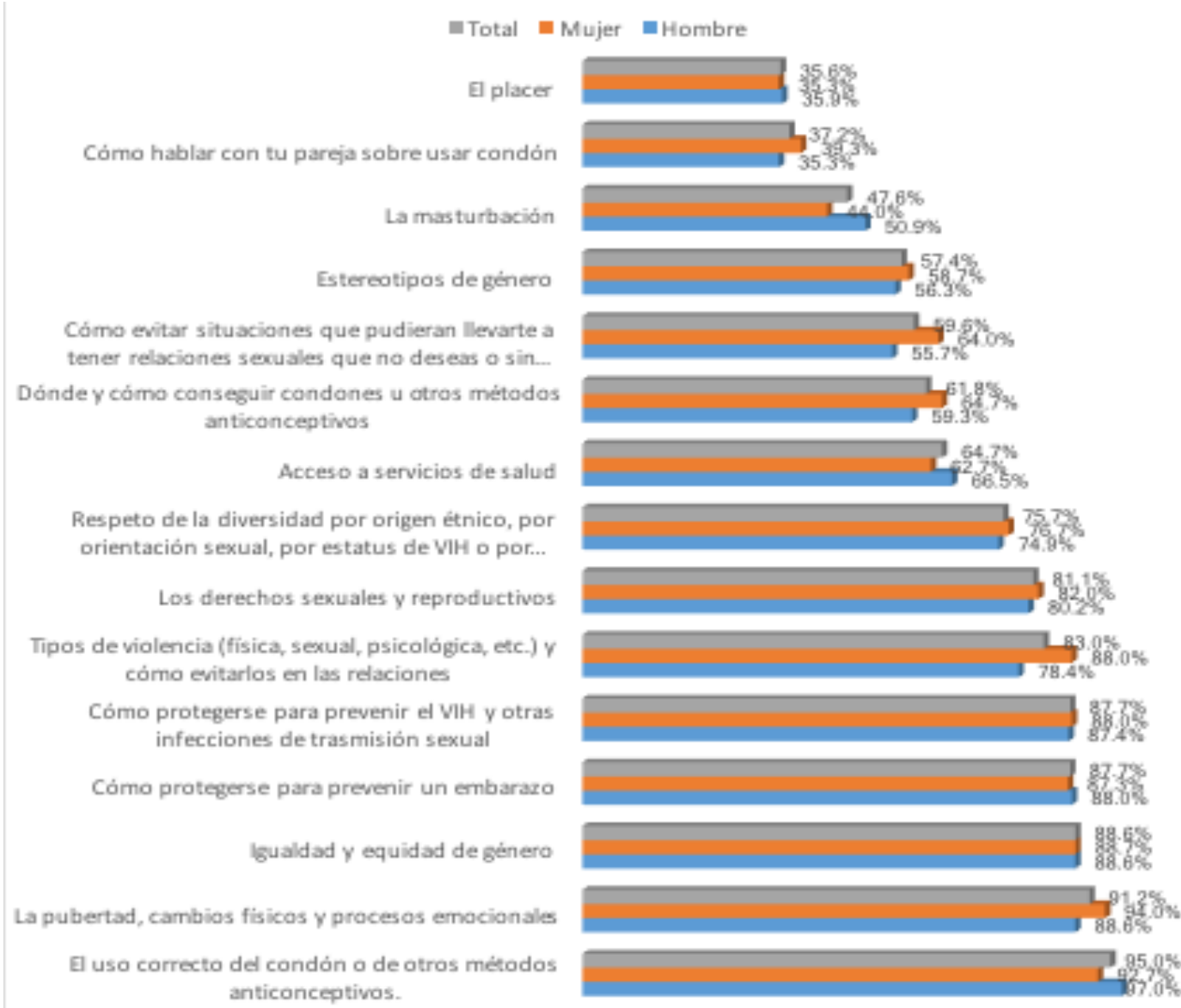

Fuente: datos de investigación propia. Querétaro, 2017.

Agrupados por dimensiones, los contenidos de ESI más abordados atañen a SSR (78.6\%), seguida de derechos y relaciones (70.2\%), y, por último, autoeficacia (63.7\%) (Gráfica 4). El estudio de Rojas et al. (2017) ya había documentado el mismo orden para estas tres dimensiones, basado en el reporte de abordaje de los temas obtenido de estudiantes de nivel medio superior. En nuestro estudio, el profesorado coincidió en que los temas de Autoeficacia son los menos atendidos en sus clases y les otorgaron el puntaje más bajo (3.2), mientras que las otras dos dimensiones obtuvieron casi el mismo promedio (Derechos y relaciones obtuvo 3.8 y SSR, 3.75) (Tabla 3). 
Gráfica 4: Abordaje reportado de las dimensiones de ESI

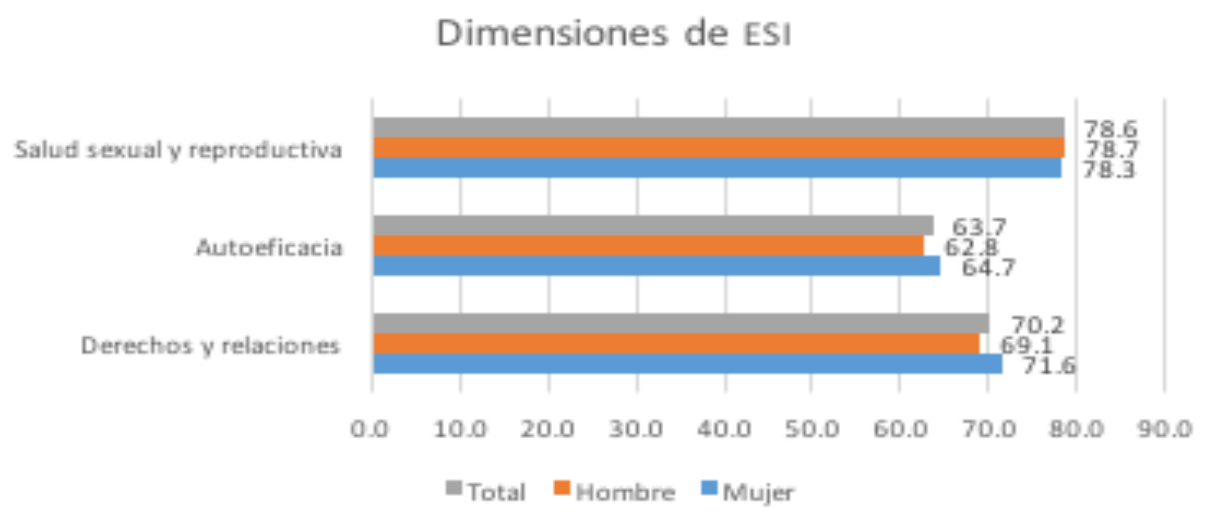

Fuente: datos de investigación propia. Querétaro, 2017.

Tabla 3: Puntajes asignados por profesorado a los temas de ESI en función de la atención que le dedican en sus clases. Querétaro, 2017

\begin{tabular}{|c|c|c|c|c|}
\hline & \multirow{2}{*}{ Temas } & \multicolumn{2}{|l|}{ Puntaje } & \multirow{2}{*}{ Promedio } \\
\hline & & Profesora & Profesor & \\
\hline 1 & El uso correcto del condón o de otros métodos anticonceptivos & 5 & 4 & $4 \cdot 5$ \\
\hline 2 & $\begin{array}{l}\text { Respeto de la diversidad por origen étnico, por orientación se- } \\
\text { xual, por estatus de VIH o por discapacidad }\end{array}$ & 5 & 5 & 5 \\
\hline 3 & Igualdad y equidad de género & 5 & 4 & $4 \cdot 5$ \\
\hline 4 & La pubertad, cambios físicos y procesos emocionales & 5 & 3 & 4 \\
\hline 5 & $\begin{array}{l}\text { Tipos de violencia (física, sexual, psicológica, etc.) y cómo evi- } \\
\text { tarlos en las relaciones }\end{array}$ & 5 & 2 & 3.5 \\
\hline 6 & Cómo protegerse para prevenir un embarazo & 5 & 5 & 5 \\
\hline 7 & $\begin{array}{l}\text { Dónde y cómo conseguir condones u otros métodos anticon- } \\
\text { ceptivos }\end{array}$ & 3 & 5 & 4 \\
\hline 8 & $\begin{array}{l}\text { Cómo protegerse para prevenir el VIH y otras infecciones de } \\
\text { trasmisión sexual }\end{array}$ & 5 & 3 & 4 \\
\hline 9 & $\begin{array}{l}\text { Cómo evitar situaciones que pudieran llevar a tener relaciones } \\
\text { sexuales que no desea o sin protección }\end{array}$ & 3 & 1 & 2 \\
\hline 10 & La masturbación & 1 & 3 & 2 \\
\hline 11 & Estereotipos de género & 5 & 2 & $3 \cdot 5$ \\
\hline 12 & Acceso a servicios de salud & 3 & 3 & 3 \\
\hline 13 & Cómo hablar con su pareja sobre usar condón & 3 & 2 & 2.5 \\
\hline 14 & El placer & 3 & 2 & 2.5 \\
\hline 15 & Los derechos sexuales y reproductivos & 5 & 3 & 4 \\
\hline \multicolumn{2}{|c|}{ Dimensión Autoeficacia } & 3.4 & 3 & 3.2 \\
\hline \multicolumn{2}{|c|}{ Dimensión Derechos y Relaciones } & 4.6 & 3 & 3.8 \\
\hline \multicolumn{2}{|c|}{ Promedio general } & 4 & 3.2 & 3.6 \\
\hline
\end{tabular}

Fuente: datos de investigación propia. Querétaro, 2017.

Diálo oos

año 11 | número 21 | julio-diciembre 2020 | ISSN 2007-2171 
Investigaciones recientes en el país han demostrado diferencias considerables en el reporte de haber recibido clases con los contenidos de ESI, entre estudiantes de secundaria y de bachillerato. Mientras que $32.9 \%$ de quienes estudian secundaria reportaron que todos los contenidos de ESI fueron abordados, solo $12.6 \%$ de quienes cursan bachillerato ofrecieron la misma respuesta (Rojas et al., 2017). En la presente investigación se obtuvo que solo $12.5 \%$ de las y los estudiantes indicó que la totalidad de los contenidos han sido retomados en sus clases, lo que coincidiría con lo documentado por jóvenes de bachillerato en el trabajo de Rojas et al. y podría ser afín con el hallazgo realizado por Carrillo et al. (2012) en Querétaro, con jóvenes de 15.8 años en promedio, en el que se destaca que solo $10 \%$ describen sus conocimientos sobre sexualidades como "muy completos", 47\% como "suficientes" y 43\% restante se distribuyó entre "regulares", "pocos" y "muy pocos". Para estudios posteriores se recomienda indagar de manera independiente los contenidos abordados en la escuela y la autopercepción de nivel de conocimiento sobre los mismos temas.

Respecto a la adquisición de métodos anticonceptivos, obtuvimos que solo $61.8 \%$ de las y los adolescentes reconoce que se les ha hablado sobre "dónde y cómo conseguir condones y otros métodos anticonceptivos". En oportunidad de su estudio, Carrillo et al. (2012) encontraron que en Querétaro las instituciones públicas de salud no estaban siendo identificadas como proveedoras principales de estos métodos. La mayoría de las adolescentes reportaban estarlos obteniendo de sus familias y los adolescentes los adquieren en las farmacias. Los rezagos en términos de acceso a servicios de salud sexual y reproductiva parecen continuar vigentes.

En cuanto al conocimiento sobre estos mismos métodos, Carrillo et al. (2012) encontraron que $90.8 \%$ de una muestra representativa de jóvenes de 15.8 años, en promedio, sabían de su existencia, siendo el condón el más identificado (92.2\%). La presente investigación confirma que, entre los contenidos de ESI que se abordan en la escuela, el reconocimiento de métodos anticonceptivos y su uso correcto está recibiendo bastante atención. 95\% de la población reportó haber escuchado sobre ello en sus clases. No obstante, en lo que respecta al manejo de información útil para prevenir las Infecciones de Transmisión Sexual (ITS) y VIH, el resultado que obtuvimos revela que en nuestra población este tema está siendo menos abordado en clases de lo que supondría el reporte de la citada investigación ( $87.7 \%$ vs. 93.4\% en la investigación de Carrillo et al. (2012). Posiblemente la diferencia se deba a que algunas personas cuentan con la posibilidad de complementar la información sobre ESI que reciben en la escuela, con otras fuentes de consulta.

Identificamos que, en general, no hay diferencia estadística en la información que han recibido hombres y mujeres, excepto que las mujeres recibieron más información que los hombres sobre los tipos de violencia (física, sexual, psicológica, etc.) y cómo evitarlos en las relaciones (78.4\% hombres vs. 88\% mujeres; $X^{2}=5.11, G L=1, p=0.02$ ). Tal parece que los mensajes que apuntan a visibilizar y evitar la violencia entre la población de nuestro estudio no han podido incorporar el 
enfoque relacional, característico de la ESI y recomendado para incidir de manera más efectiva en la prevención de la violencia y otras problemáticas asociadas (Rojas et al., 2017). Dicho enfoque hace posible que tanto las mujeres como los hombres se orienten a la construcción de relaciones horizontales y tengan herramientas para el manejo no violento de conflictos. Esto es especialmente prioritario en el caso de Querétaro, que encabeza las estadísticas más recientes de violencia contra las mujeres a nivel nacional, aportadas por la Encuesta Nacional sobre la Dinámica de las Relaciones en los Hogares (Endireh) $2016^{8}$ (Instituto Nacional de Estadística y Geografía - INEGI), 2017).

Sin aspiraciones concluyentes, dado el reducido tamaño de la muestra de docentes con quienes se trabajó, se puede señalar que se esbozaron algunas diferencias en la profundidad con que la profesora y el profesor responsables de la ESI en este plantel, abordan los diferentes contenidos que integran la materia. Estas diferencias podrían estar relacionadas con sus propias representaciones e identidades de género (en tanto son compatibles con roles y atribuciones tradicionales sobre ser hombre y ser mujer). Por ejemplo, el tema que recibe menos atención en las clases impartidas por la profesora es la masturbación, mientras que su colega varón no aborda con demasiado ahínco tópicos como: la violencia, cómo evitar situaciones que pudieran derivar en relaciones sexuales indeseadas o desprotegidas, estereotipos de género, cómo hablar con tu pareja sobre usar condón y el placer. La ESI no es solo un contenido académico, es una caja de herramientas para la vida que no puede explorarse a cabalidad, ni trasmitirse, si el personal docente comisionado a dicha tarea no ha tenido la posibilidad de apropiarse plena y vivencialmente de sus componentes (SEP, 2012). Investigaciones posteriores podrían profundizar en este campo, para contribuir al fortalecimiento de la labor docente en materia de ESI.

Globalmente, nuestros resultados son consecuentes con la evaluación de la Declaración Ministerial Prevenir con Educación Pública, realizada en 2015, donde se identificó que la ESI en México es particularmente deficiente en cuanto a temas de género, relaciones interpersonales y placer (Save the Children, 2016: 16).

\section{Reflexiones finales}

Un pequeño grupo de la población de nuestro estudio (4\%) desea tener hijos/as antes de los 20 años, y en ningún caso, quienes lo integran reportaron que todos los contenidos de ESI hayan sido retomados en sus clases escolares. A corto plazo, su deseo podría transformarse en un acto, pero difícilmente contará con bases para desplegarse como una decisión informada. El resto de la muestra tampoco está en una posición aventajada en términos de haber recibido ESI, de modo que, si sus expectativas acompasan su devenir, podrían llegar a reproducirse sin

8 En la demarcación, casi tres cuartas partes (71.2\%) de las mujeres de 15 años y más, han vivido al menos un episodio de violencia a lo largo de sus vidas y prácticamente una tercera parte (32.5\%) ha vivido violencia escolar, mientras que la media nacional en este ámbito es de 25.3\% (INEGI, 2017). 
acrecentar las estadísticas de EA (o incluso podrían no reproducirse), pero tampoco cuentan con suficiente información para evitar prácticas sexuales de riesgo, embarazos no deseados o situaciones de violencia, que no pierden el potencial de trastocar negativamente su existencia por el hecho de presentarse en etapas más avanzadas de su vida.

Identificamos sesgos y diferencias estadísticas en razón de género, compatibles con estereotipos tradicionales. Los hombres se mostraron más proclives a la reproducción en general y también a la reproducción a temprana edad. Reciben menos información (o son menos receptivos) cuando se habla sobre la tipología de la violencia y sobre cómo evitar relaciones sexuales no deseadas o desprotegidas. Además, como padres de mujeres adolescentes, su presencia es casi nula en la consideración de sus hijas como principales informantes cuando ellas tienen dudas sobre temas de sexualidades.

Por su parte, las mujeres desean postergar su reproducción hasta una edad un poco más avanzada que los varones y casi $30 \%$ de ellas indicó que la maternidad no está entre sus planes futuros. No obstante, a pesar de que en la mayoría de los temas de ESI las jóvenes obtuvieron resultados muy similares a los de sus pares (incluso con un porcentaje superior en algunos tópicos), ellas están menos expuestas a recibir información sobre la masturbación y por lo tanto, más lejanas a la posibilidad de conocer su propio cuerpo y acceder a la facultad subjetiva de desligar el placer de la reproducción. ${ }^{9}$ Esto puede constituir para ellas un factor de riesgo frente al EA (Echeverría-Lozano, 2017). Finalmente, las mujeres en su rol de madres de adolescentes son reconocidas por sus hijas e hijos como su fuente de mayor confianza cuando se trata de consultas sobre sexualidades.

Para nuestra población de estudio, como para el resto del país y del mundo, la igualdad no ha logrado pasar todavía del plano formal al de hecho (Carrillo et al., 2012). Si en el mejor de los casos, los hombres y las mujeres estuviesen expuestos/as a los mismos contenidos de ESI, su pertenencia a una cultura que los desiguala hace necesario que se redoblen esfuerzos para que unos y otras incorporen aquellos temas que tradicionalmente les son menos asequibles por su adscripción de género.

Falta mucho camino por avanzar para que los y las adolescentes puedan ejercer los derechos sexuales y los derechos reproductivos que deberían estarles garantizados. Los servicios de salud no son plenamente identificados por ellas y ellos como fuentes de información o proveedores de métodos anticonceptivos gratuitos; y aunque sus familias y escuela estén realizando esfuerzos muy loables por tratar temas relacionados con la ESI, las metodologías podrían no estar facilitando la apropiación significativa de los mismos y los contenidos quizá no estén logrando

9 Tradicionalmente, las mujeres "no han sido pensadas como sujetos sexuados, mucho menos se les ha considerado como aptas para conocer su cuerpo y por tanto otros cuerpos..." (SEP, 2012: 92). Además, está presente "el mandato social de no sentir, que se ve reflejado en la nula experimentación del placer por medio de la masturbación como una opción para satisfacer el deseo sexual, placer del que ningún otro(a) sea responsable" (Echeverría-Lozano, 2017: 48).

Diálo pos 
trasmitirse desde una visión plenamente científica y no atravesada por estereotipos de género. Investigaciones posteriores en el contexto local podrían confirmar estas condiciones ya documentadas por estudios previos (Rojas et al., 2017; SEP, 2012).

Aunque se anuncia que las múltiples tensiones políticas que atañen al tema —por la imbricación de intereses y preceptos religiosos - no cesarán a corto plazo, la laxitud y la permisividad del Estado en esta materia no solo afecta a las y los jóvenes; sino que también puede convertirse en un potencial foco de conflictividad entre las instituciones educativas y las familias, en lugar de aportarles bases firmes para construir colectivamente diálogos y estrategias con posibilidad de disentir, pero no de negociar (CDHDF, 2016). Porque los derechos no se negocian.

\section{Agradecimientos}

Esta investigación fue desarrollada en el marco de un proyecto encaminado a promover entre adolescentes el desarrollo de factores de protección frente a conductas y situaciones de riesgo relacionadas con la sexualidad, apoyado con recursos del Instituto Nacional de las Mujeres, a través de la Décima Sexta Emisión del Programa Proequidad. Agradecemos haber tenido la oportunidad de participar desde nuestro saber y experiencia, de la mano del gobierno, en la construcción de estrategias para repensar, afrontar y resolver los problemas de nuestro país.

\section{Referencias bibliográficas}

Altamirano, C. (2017). Falta de oportunidades y búsqueda de amor, las causas del embarazo adolescente en México. Animal Político. http://www.animalpolitico.com/2017/11/embarazo-adolescente-mexico-casos/

Amuchástegui, A.; E. Flores; E. Aldaz (2015). Disputa social y disputa subjetiva. Religión, género y discursos sociales en la legalización del aborto en México. La ventana, 1(41), 153-195. http://www.scielo.org.mx/scielo.php?script=sci arttext\&pid=S1405-94362015000100153

Atienzo, E.; L. Campero; A. L. Lozada; C. Herrera (2014). Aspiraciones educativas y familiares como condicionantes en la prevención de embarazos tempranos en México. Salud Pública de México, 56(3), 286-294. https://doi.org/10.21149/spm.v56i3.7347

Carrillo, M. A.; M. A. Mendoza; J. R. Salinas; J. J. Martínez; A. M. Carrillo; M. L. Luján; A. Ribón (2012). Estudio sobre atención y prevención del embarazo en adolescentes de los programas que operan las dependencias responsables del Estado de Querétaro. Querétaro. http://cedoc. inmujeres.gob.mx/ftpg/Qro/QRO MA1 ESTUDIO 12.pdf 
Comisión de Derechos Humanos del Distrito Federal (2016). Informe anual 2015, Situación de los derechos humanos de las mujeres, embarazo en adolescentes y el ejercicio de los derechos sexuales y reproductivos en la Ciudad de México. Ciudad de México: Autor.

Congreso General de los Estados Unidos Mexicanos (2014). Ley General de los Derechos de niñas, niños y adolescentes. Diario Oficial de la Federación. http://www.dof.gob.mx/nota detalle.php?codigo $=5374143 \&$ fecha $=04 / 12 / 2014$

Consejo Nacional de Población (2016). Situación de la Salud Sexual y Reproductiva, República Mexicana. https://www.gob.mx/cms/uploads/attachment/file/237216/Cuadernillo SSR RM.pdf

Díaz, A. J.; L. G. Ayala; L. J. Sandoval; H. H. Rocha; Y. C. Aguirre; A. Soto (2018). Manual de capacitación para la incorporación de los hombres en la prevención de embarazo en adolescentes desde la perspectiva de género. México: INMUJERES. http://cedoc.inmujeres.gob.mx/documentos download/101303.pdf

Echeverría-Lozano, A. (2017). Deseo sexual en jóvenes de la Ciudad de México: amor vs. placer. Journal of Behavior, Health \& Social Issues, 9(2), 45-53. https://doi.org/10.1016/j.jbhsi.2017.10.001

Family Care International (2008). Buenas prácticas en materia de prevención del embarazo adolescente. http://www.orasconhu.org/sites/default/files/Oct 08 Concepto BP en PEA -4.pdf

Fernández, A. M. (1998). Por una mejor atención a las niñas-madres. Revista Subjetividad y Cultura, 11, 1-14. http://subjetividadycultura.org.mx/por-una-mejor-atencion-a-las-ninas-madres/

Fernández, A. M. (2013). Jóvenes de vidas grises: psicoanálisis y biopolíticas. Buenos Aires: Nueva Visión.

Figueroa-Perea, J. G. (2005). Algunos dilemas éticos y políticos al tratar de definir los derechos reproductivos en la experiencia de los varones. Perspectivas Bioéticas, 1-16. http://menengage.org/wp-content/uploads/2014/06/Dilemas eticos y politicos derechos reproductivos varones.pdf

Figueroa-Perea, J. G.; V. Sánchez (2000). La presencia de los varones en el discurso y en la práctica del aborto. Papeles de Población, 59-82. http://www.redalyc.org/articulo.oa?id=11202504

Fondo de Población de las Naciones Unidas (2013). Maternidad en la niñez. El estado de la población mundial 2013. Nueva York: UNFPA. https://www.unfpa.org/sites/default/files/pub-pdf/ ES-SWOP2013.pdf

Fondo de Población de las Naciones Unidas (2017). Mundos aparte: La salud y los derechos reproductivos en tiempos de desigualdad. Estado de la población mundial 2017. Nueva York: UNFPA. http://www.unfpa.org/sites/default/files/sowp/downloads/UNFPA PUB 2017 ES SWOP Estado de la Poblacion Mundial.pdf

García-Hernández, G. E. (2014). Embarazo adolescente y pobreza, una relación compleja. Iztapalapa Revista de Ciencias Sociales y Humanidades, 77, 13-53. https://dialnet.unirioja.es/descarga/articulo/5716864.pdf 
Instituto Mexicano para la Competitividad (2017). Mejora tu escuela. http://www.mejoratuescuela.org/

Instituto Nacional de Estadística y Geografía (2017). Resultados de la Encuesta Nacional sobre la Dinámica de las Relaciones en los Hogares (Endireh) 2016. Boletín de Prensa Núm. 379/17. México: INEGI. http://www.inegi.org.mx/saladeprensa/boletines/2017/endireh/endireh2017 08.pdf

Instituto Nacional de Salud Pública (2015). Estudio sobre la prevención del embarazo en adolescentes desde las masculinidades. Informe final. http://cedoc.inmujeres.gob.mx/documentos download/Estudio-ENAPEA.pdf

Lagarde, M. (2015). Los cautiverios de las mujeres: madresposas, monjas, putas, presas y locas. Ciudad de México: Siglo XXI.

Ochoa, I. M. (2018). Sólo nueve secundarias con educación sexual explícita. Diario de Querétaro. https://www.diariodequeretaro.com.mx/local/solo-nueve-secundarias-con-educacionsexual-explicita-1871393.html

Pacheco-Sánchez, C. I. (2016). Embarazo en menores de quince años: Los motivos y la redefinición del curso de vida. Salud Pública de México, 58(1), 56-61. https://doi.org/10.21149/spm. v58i1.7668

Perrotta, G. (2018). Embarazo y maternidad en la adolescencia. Revista Electrónica de la Facultad de Psicología UBA, 27. http://intersecciones.psi.uba.ar/index.php?option=com content\&vi ew=article\&id=702:embarazo-y-maternidad-en-la-adolescencia\&catid=9:perspectivas\&lte $\underline{\mathrm{mid}=1}$

Poder Ejecutivo del Estado de Querétaro (2019). AGEBS del Estado de Querétaro. http://www. enlacemunicipaldequeretaro.gob.mx/Portals/O/Oficios/2015/SEDESOL/Oficio 1684-2015/ AGEBS ESTADO DE QRO.xIsX

Posada, C. (2014). Embarazo en la adolescencia: no una opción, sino una falta de opciones. Revista Sexología y Sociedad, 25, 4-10. http://revsexologiaysociedad.sld.cu/index.php/sexologiaysociedad/article/view/268/327

Quiroz, J.; E. E. Atienzo; L. Campero; L. Suárez-López (2014). Entre contradicciones y riesgos: Opiniones de varones adolescentes mexicanos sobre el embarazo temprano y su asociación con el comportamiento sexual. Salud Pública de México, 56(2), 180-188. http://www.scielo. org.mx/scielo.php?script=sci arttext\&pid=S0036-36342014000200005\&lng=es\&tlng=es

Rocha-Sánchez, T. (2015). Entre el techo y las fronteras de cristal en Latinoamérica: Retos y vicisitudes vigentes en el proceso de empoderamiento de las mujeres. Entorno, 0(54), 32-41. http://biblioteca.utec.edu.sv/entorno/index.php/entorno/article/view/25/26

Rojas, R.; F. de Castro; A. Villalobos; B. Allen-Leigh; M. Romero; A. Braverman-Bronstein; P. Uribe (2017). Educación sexual integral: Cobertura, homogeneidad, integralidad y continuidad en escuelas de México. Salud Pública de México, 59(1), 19-27. https://doi.org/10.21149/8411 
Rosales-Mendoza, A. L.; F. Salinas-Quiroz (2017). Educación sexual y género en primarias mexicanas ¿qué dicen los libros de texto y el profesorado? Revista Electrónica Educare, 21(2), 1-21. https://doi.org/http://dx.doi.org/10.15359/ree.21-2.11

Save the Children. (2016). Embarazo y maternidad en la adolescencia. México. https://www. savethechildren.mx/sci-mx/files/89/89cd3f0a-43e0-42aa-876a-a345df7f0f74.pdf

Secretaría de Educación Pública (2012). Embarazo adolescente y madres jóvenes en México: una visión desde el Promajoven. México. https://doi.org/9786079200633

Stern, C. (2003). Significado e implicaciones del embarazo adolescente en distintos contextos socioculturales de México: reseña de un proyecto en proceso. Estudios Sociológicos, 21(63), 725-745. https://doi.org/10.2307/40420800 